Instituto Internacional de Investigación y Desarrollo Tecnológico Educativo INDTEC, C.A.

DOI: https://doi.org/10.29394/scientific.issn.2542-2987.2017.2.6.3.52-73

OAI-PMH: http://www.indteca.com/ojs/index.php/Revista Scientific/oai

\title{
La Educación Ecuatoriana en Inglés: Nivel de Dominio y Competencias Lingüísticas de los Estudiantes Rurales
}

\author{
Autores: Diego Patricio Ortega Auquilla \\ Universidad Nacional de Educación, UNAE \\ dipaortega@gmail.com \\ Cuenca, Ecuador \\ Roxana Auccahuallpa Fernández \\ Universidad Nacional de Educación, UNAE \\ roxana.auccahuallpa@unae.edu.ec \\ Cuenca, Ecuador
}

\section{Resumen}

La enseñanza-aprendizaje del inglés ha dado un giro grande en el sistema educativo ecuatoriano en las últimas dos décadas y varias medidas han sido implementadas. En este sentido, exámenes internacionales y estandarizados se han convertido en los medios que acreditan la suficiencia de esta lengua extranjera de estudiantes y profesores de distintos niveles educativos. Es por ello que el presente estudio tuvo como objetivo determinar el nivel de suficiencia de inglés de los estudiantes en el último año de Bachillerato General Unificado (BGU), como también identificar en qué competencias lingüísticas los estudiantes presentan mayores y menores dificultades. Para alcanzar estos objetivos, se llevó a cabo un estudio cuantitativo de tipo exploratorio en ocho instituciones educativas fiscales rurales del cantón Azogues en Ecuador. Los resultados del test, aplicada a una muestra de 142 estudiantes, indican que el nivel de suficiencia de inglés de los participantes es insatisfactorio, corroborando datos existentes sobre el bajo nivel de suficiencia de inglés en el estudiantado Ecuatoriano. Además, los participantes mostraron un bajo nivel de dominio en tres competencias lingüísticas, lo cual dificultaría el uso del idioma meta durante diferentes propósitos en su vida futura.

Palabras clave: enseñanza secundaria; investigación educativa; lengua extranjera; inglés. 


\section{Ecuadorian English Education: Level of Proficiency and Linguistic Competences of Rural Students}

Date Received: 01-07-2017

Date Acceptance: 30-08-2017 


\section{Introducción}

En el Ecuador, el inglés es el idioma extranjero que oficialmente se enseña en establecimientos educativos privados y públicos a nivel nacional (Avila, 2010). En este sentido, el Ministerio de Educación del Ecuador (de aquí en adelante MinEduc), ha implementado varias medidas enfocadas a mejorar la enseñanza-aprendizaje de la lengua inglesa en las instituciones educativas. Una de las primeras medidas adoptadas ha sido la obligatoriedad de la enseñanza del inglés en la educación secundaria ecuatoriana desde el año 1992 (British Council, 2015). En 1992, por medio de un convenio entre el MinEduc y el British Council una importante reforma curricular para la asignatura de Inglés fue llevada a cabo para las instituciones educativas públicas y fiscomisionales. Esta acción realizada por el gobierno ecuatoriano a través del MinEduc, con apoyo foráneo, puede ser entendida como una de las iniciativas gubernamentales más importantes para mejorar el proceso de enseñanza-aprendizaje del idioma inglés en el sistema educativo del país.

La reforma curricular en el área de inglés liderada por el MinEduc y el British Council a través del proyecto denominado Reforma Curricular para el Desarrollo del Aprendizaje de Inglés o Curriculum Reform and Development for the Learning of English (CRADLE), fue aplicado a los colegios a nivel nacional (Calle et al., 2012). Este proyecto incluyó la creación de una serie de seis textos denominados Our World Through English. Las lecciones de estos textos presentaban a los estudiantes la realidad del país por medio de la lengua inglesa. La serie de textos incluía guías didácticas, material auditivo y material de exámenes. Estos materiales para la asignatura de inglés fueron utilizados aproximadamente por 1.200.000 estudiantes y sus profesores en los años escolares o electivos de 1993-1994 a 1998-1999 (MinEduc, 2009). Sin embargo, a pesar de las innovaciones que formaban parte de esta reforma curricular por medio del proyecto CRADLE, el bajo rendimiento de los estudiantes de inglés ha sido evidente. Problema que se refleja al momento 
que los estudiantes ingresan a la universidad, ya que ellos no logran acceder a niveles intermedios o avanzados de inglés (Calle et al., 2012).

Luego el proyecto Fortalecimiento de la Enseñanza del Inglés promovido nuevamente por el MinEduc entro en vigencia. Este proyecto pretende mejorar las competencias lingüísticas de los profesores de inglés como también su práctica docente. Entre los objetivos generales del proyecto se puede citar lo siguiente: la implementación de un currículo nuevo de inglés que responde a los lineamientos establecidos en el Marco Común Europeo de Referencia para la Enseñanza, Aprendizaje, y Evaluación de Lenguas Extranjeras (MCER), la distribución gratuita de textos de inglés alineados al currículo nuevo, la creación e implementación de un sistema nuevo de evaluación basado en pruebas estandarizadas para los profesores de inglés en ejercicio docente y, también, para aquellos que desean ingresar al sector educativo público, y la organización de un sistema de desarrollo profesional permanente, lo cual incluía capacitaciones afuera del país como el programa Go Teacher (MinEduc, 2016).

En suma, a pesar de los esfuerzos realizados desde los años noventa, únicamente un mínimo de estudiantes del Bachillerato General Unificado (BGU) logran alcanzar los niveles deseados de suficiencia del idioma inglés al culminar sus estudios de secundaria. Así mismo, la mayoría de los estudiantes universitarios presentan problemas para lograr obtener la acreditación de suficiencia respectiva, según el MCER, lo cual es un requisito actual de graduación (El Comercio, 2016). Esta problemática también se refleja en la dificultad que los estudiantes tienen para acceder a becas internacionales o inclusive para continuar sus estudios en posgrados. Es por ello que este estudio proporciona evidencia empírica sobre el nivel de suficiencia de los estudiantes del tercer año de bachillerato de ocho instituciones educativas fiscales de las parroquias rurales del cantón Azogues en Ecuador. Las preguntas de investigación que sirvieron de guía en el presente estudio son 
las siguientes: ¿Cuál es el nivel de dominio de los estudiantes de inglés de los terceros años de bachillerato de las parroquias rurales del cantón Azogues en relación al nivel establecido en las Directrices Curriculares Nacionales de Inglés (DNCl) y el Currículo de Inglés vigente? y ¿Cuál es la competencia lingüística o competencias lingüísticas en la que los estudiantes tienen mayores y menores dificultades?

\section{Consideraciones importantes sobre la enseñanza-aprendizaje del inglés en el Ecuador.}

En esta sección se examinan temas que tienen una estrecha relación con la educación en inglés en el Ecuador y el análisis de estos temas representan la génesis del presente estudio, el cual intenta aportar a la discusión vigente sobre el bajo nivel de suficiencia de inglés en el estudiantado ecuatoriano.

\subsection{Directrices curriculares nacionales de inglés}

Las Directrices Curriculares Nacionales de Inglés (DCNI) para el BGU, elaborado a raíz de la creación del Proyecto de Fortalecimiento de la Enseñanza del inglés, estuvieron vigentes desde el año 2012 hasta el 2016 y presentaron algunas características fundamentales. Estas directrices fueron de gran importancia en la educación en inglés a nivel nacional y en base de las mismas se forjo el Currículo 2016-2017 de Inglés; las DCNI y el Currículo de Inglés actual poseen características similares. En este sentido, el MCER y una enseñanza comunicativa del inglés son evidentes en ambas iniciativas curriculares. Por primera vez las DCNI estableció que los estudiantes que culminen el Bachillerato deben alcanzar un nivel B1 según el MCER - lo cual es el objetivo general de este tipo de directrices. Las características principales de las DCNI tienen estrecha relación con el enfoque de Enseñanza Comunicativa de Lenguas (Communicative Language Teaching/ CLT como se 
le conoce en inglés) y el MCER. Es relevante mencionar que los lineamientos curriculares de inglés fueron forjados en base del MCER y que el CLT constituye la filosofía fundamental en la que las directrices o lineamientos están sustentados (MineEduc, 2012).

El enfoque de Enseñanza Comunicativa de Lenguas (o CLT) antes mencionado ha sido utilizado a nivel mundial debido a que incluye una serie de principios acerca de la naturaleza del idioma y la enseñanza y aprendizaje de lenguas, con especial énfasis en la autenticidad de la lengua meta (es decir la lengua que los estudiantes o personas tratan de adquirir). El mismo propone organizar un silabo en base a diferentes funciones de la lengua extranjera funciones (e.g. preguntar direcciones, solicitar permiso, realizar una invitación, pedir disculpas) que los estudiantes necesitan dominar para poder interactuar en inglés efectivamente. Mientras que el MCER proporciona una base común para la elaboración de lineamientos curriculares y sílabos para la enseñanza y aprendizaje de lenguas. Este marco de referencia ha tenido un papel importante en políticas lingüísticas a nivel internacional debido a que guía el proceso de enseñanza-aprendizaje de un segundo idioma (o lenguas extranjeras) en sistemas educativos. Específicamente, la importancia del marco de referencia se centra en la guía que proporciona a los programas y profesores de lenguas extranjeras para ayudar a los aprendices a alcanzar suficiencia en la lengua meta. Es decir, este marco traza un camino que determina lo que los estudiantes deben poder hacer en las diferentes etapas del proceso de aprendizaje dentro de los seis niveles establecidos (A1, A2, B1, $\mathrm{B} 2, \mathrm{C} 1$ y C2) y que conocimientos y destrezas deben ser adquiridos para comunicarse efectivamente en la lengua meta (Ureña, 2014; MinEduc, 2012).

En 2012, el MinEduc hizo público varios documentos con el objetivo de brindar información que ayude a implementar las orientaciones curriculares y pedagógicas que constituyen las DCNI de manera efectiva en las aulas del BGU. Estos documentos oficiales y claves tienen los siguientes títulos: 
National Curriculum Specifications, Planning Guidelines, y Ecuadorian inservice English Teacher Standards: The English Language Learning Standards. Las nociones que un idioma es un sistema para la expresión y transferencia de significado como también que un idioma es una herramienta prioritariamente de interacción y comunicación son reflejadas en estos documentos, especialmente en el National Curriculum Guidelines: English as a Foreign Language. Este tipo de nociones deben ser llevadas a la práctica docente a través de una instrucción basada en el enfoque de Enseñanza Comunicativa de Lenguas (British Council, 2015).

Por otra parte, en la educación rural y urbana la obtención del nivel de inglés esperado por parte de los estudiantes que culminan la secundaria debe ser promovida por los profesores quienes tienen el deber de implementar un enfoque comunicativo-funcional del idioma meta en su práctica docente, según lo dispuesto en las DCNI. Además de basarse en los propósitos comunicativos $y$ en las distintas funciones de la lengua inglesa al momento de planificar e implementar la enseñanza de este idioma, los profesores deben conocer acerca del MCER minuciosamente y las metodologías de enseñanza contemporáneas (e.g., CLIL y TBLT) de idioma extranjero. Si los profesores fundamentan efectivamente su práctica docente en el enfoque comunicativofuncional, descrito en las DCNI, sus estudiantes al terminar el BGU deberían alcanzar el nivel de suficiencia de inglés requerido. Así que conocer si la aplicación de las DCNI se está llevando a cabo en el sistema educativo ecuatoriano es una temática que no ha sido abordaba anteriormente y merece atención especial y urgente.

\subsection{Situación del nivel de inglés en la educación secundaria del Ecuador}

Las instituciones educativas del área rural de Ecuador han sido aquellas que históricamente han presentado mayores desventajas en las distintas facetas del sistema educativo, es decir, en cuanto a recursos económicos y 
humanos, logística y preparación. Por ejemplo, un estudio a profundidad elaborado por Alejandra Calderón, mostró que la tasa de analfabetismo en el área rural triplica la del área urbana, el promedio de años de estudio es menor en dos años, y el acceso a internet es de apenas $2,41 \%$, casi 7 puntos porcentuales por debajo del área urbana (Calderón, 2015a).

Consecuentemente, la educación en la asignatura de inglés en la ruralidad adolece de los problemas más graves e imaginables pues no cuentan con los recursos económicos, humanos y materiales necesarios para el aprendizaje de este idioma y en muchos de los casos ni siquiera se enseña inglés. Las investigaciones que se han realizado sobre la educación rural todavía son escasas y los indicadores no se han trabajado a profundidad (Calderón, 2015b), por lo que no puede hablarse todavía de los problemas de la enseñanza del idioma con completa claridad.

A pesar de la serie de acciones y medidas implementadas a nivel nacional para mejorar la adquisición de la lengua inglesa de los estudiantes ecuatorianos, el nivel suficiencia de este idioma no es satisfactorio en la población. La empresa internacional especializada en la enseñanza de lenguas Education First realizó una tercera edición del estudio titulado Índice del Nivel de Inglés, en el cual se dio a conocer que el Ecuador se ubicó en el puesto 48 de 60 países con un puntaje de 46.90 sobre 100. Este ranking evidenció que Ecuador descendió dos lugares en relación con los resultados del estudio realizado en el 2012 (El Telégrafo, 2014b). En el 2015, se realizó una nueva versión del estudio antes mencionado; el reporte indicó que el Ecuador se volvió a ubicar en el grupo de países que poseen un nivel bajo de suficiencia de inglés a nivel mundial (Education First, 2015).

Por otro lado, Ureña (2014), ha manifestado que se debe tener en cuenta el nivel de suficiencia de inglés requerido a los profesores del sector público, quienes deben poseer un nivel B2 según el MCER. Esto indica que los profesores de inglés solo necesitan ser usuarios independientes, de nivel 
intermedio alto, del idioma que enseñan, cuando deberían tener un dominio por encima de esto. Es decir, deberían tener al menos un nivel C1, lo cual indica que son usuarios competentes con dominio operativo eficaz del idioma. Esto sería importante si se pretende que los estudiantes logren el nivel requerido por el MinEduc. Autores como Staehr y Kuhlman (2010), sostienen que el dominio de la lengua inglesa es un requisito profesional que los profesores de este idioma deben cumplir.

\subsection{Posibles causas de los resultados del aprendizaje del idioma inglés}

Algunas de las causas que se infieren, sobre la problemática planteada anteriormente, tienen que ver con el nivel de preparación de los docentes que enseñan inglés en el sistema de educación secundaria en el país. Por consiguiente, se han realizado esfuerzos por mejorar, a tal punto que fueron alrededor de 791 docentes los que se beneficiaron del programa Go Teachers; algunos de las cohortes de este programa estudiaron en la Universidad del Estado de Kansas por un lapso de 7 meses (El Telégrafo, 2014a). Además, alrededor de 2.500 docentes a nivel nacional fueron capacitados para obtener su certificación B2 que les garantice ser usuarios independientes del idioma (El Ciudadano, 2015). No obstante, esto solo representa el $12 \%$ de los docentes de inglés que necesita el país (El Ciudadano, 2015); es por ello que los estudios realizados sobre el nivel de dominio del idioma inglés de los docentes a nivel nacional son insatisfactorios y las estadísticas son preocupantes sobre el bajo nivel de suficiencia de la población estudiantil y población en general. A pesar de todo esto, el nivel de dominio actual del idioma inglés de los docentes no es la única causa de los resultados de aprendizaje insatisfactorios de la lengua meta de los estudiantes de secundaria.

Por ejemplo, otros factores están relacionados con el contexto social ecuatoriano. Lo que aprenden los estudiantes dentro del sistema escolar no 
es replicable en el contacto social tanto con la familia como con el resto de amigos o familiares porque el idioma en el que se comunican a diario no es el inglés. En otras palabras, el estudiante no encuentra con quién hablar inglés fuera de la escuela o por lo menos no puede practicar lo aprendido, lo que sí ocurre con la gran mayoría de conocimientos que se fortalecen justamente en su contacto con la sociedad para convertirse en aprendizajes más significativos. Algunas instituciones educativas han solventado este problema mediante el uso de plataformas informáticas que permiten el contacto estudiante-docente en horas que están fuera de clase. Otros colegios en cambio han establecido que materias como Estudios Sociales o Ciencias Naturales se impartan en inglés logrando aterrizar en algo concreto lo que aprenden. Por último, algunas instituciones han optado por aumentar el número de horas y otorgar oportunidades de intercambio con colegios en el extranjero. Sin embargo, es claro que esta solución no es factible para todas las instituciones educativas, especialmente para el sector público.

Adicionalmente, una enseñanza ineficaz puede tener efectos negativos en la clase de inglés. Esto se evidenció en una investigación llevada a cabo en las instituciones educativas públicos de la ciudad de Cuenca en el marco del currículo de inglés anterior, a cargo del proyecto CRADLE. Específicamente, este estudio examinó las prácticas docentes de los profesores de inglés del sector público; los resultados indicaron que varios aspectos afectan al conocimiento y habilidades de los estudiantes de inglés de educación secundaria. Los factores que dificultan que los estudiantes de secundaria posean un conocimiento adecuado de la lengua inglesa $y$, consecuentemente, puedan comunicarse en esta lengua se relacionan con el uso de una metodología de enseñanza tradicionalista. Esta metodología implica un aprendizaje centrado en el profesor, como también la falta de interacción entre los estudiantes en la lengua inglesa. Otro factor es la 
confusión que poseen los profesores al intentar implementar estrategias comunicativas en el aula de clase (Calle et al., 2012).

\section{Métodos}

\subsection{Materiales}

Esta investigación explora el cumplimento del objetivo general de las DNCl; este mismo objetivo también debe ser alcanzado a través de la implementación del Currículo de Inglés actual - el cual sostiene que los estudiantes al culminar la educación secundaria deben lograr un nivel B1 según el MCER.

Para la investigación se llevó a cabo un estudio cuantitativo de tipo exploratorio. Para Creswell (2009) y Hernández, Fernández y Baptista (2014) este método utiliza la recolección de datos para examinar un tema educativo poco estudiado, todo con el fin de establecer la realidad educativa. La metodología exploratoria permite identificar aquellos componentes de un fenómeno educativo a fin de determinar la situación real en el cuál se encuentran los estudiantes de tercero de bachillerato de las parroquias rurales del Cantón Azogues con relación a su nivel de dominio del idioma inglés.

Por lo tanto, la población del estudio corresponde a 272 estudiantes que estuvieron matriculados en el tercero de bachillerato de instituciones educativas públicas de las ocho parroquias rurales del Cantón Azogues de la provincia del Cañar en Ecuador durante el año electivo 2016-2017. Se seleccionó una muestra de 160 estudiantes con un 95\% de confiabilidad. De los cuales, 142 estudiantes entre féminas y varones de 16 y 18 años participaron en el estudio que corresponde al $88.75 \%$, es decir, a este número de estudiantes fue administrado un test de inglés. Cuatro estudiantes no dieron información propicia y no se les pudo identificar para los resultados del estudio.

Se obtuvo la debida aprobación de parte de las autoridades de las instituciones educativas y los padres de familia de los participantes menores 
de edad; esto es importante debido a que "existe todo un conjunto o una serie de derechos que no deben ser violados con el fin de que permanezca: la convivencia, honradez, dignidad, privacidad y sensibilidad. Así como también, la integridad en las instituciones escolares..." (Abreu, 2017, pág. 340).

Los instrumentos para la recolección de datos fueron el test PET (Preliminary English Test), el cual fue administrada por el Instituto de Idiomas de la Universidad de Fuerzas Armadas ESPE ubicada en la ciudad de Quito; esta universidad es categorizada como A según el ranking de universidades en Ecuador. Además, el Instituto de Idiomas cuenta con la acreditación del ETS (Educational Testing Services) como centro autorizado para la recepción del examen TOEFL. Es por ello que la Universidad Nacional de Educación (UNAE), a través de gestiones del Vicerrectorado Académico, encargó al instituto de idiomas de la ESPE la administración del test. Los acuerdos entre la UNAE y la ESPE fueron establecidos por parte de la UNAE, quien pagó los viáticos del equipo de trabajo conformado por cuatro profesores de la ESPE, quienes realizaron todo el proceso de preparación y administración del test y, posteriormente, entregaron los resultados.

El test fue aplicado en noviembre del 2016 a la muestra de estudiantes en dos días diferentes. El test tuvo como propósito examinar el dominio de los estudiantes sobre las competencias lingüísticas de inglés. La administración del test fue en dos lugares estratégicos de acuerdo con la disponibilidad de los participantes del estudio. (1) Cinco instituciones educativas completaron el test en la Universidad Católica de Cuenca - sede Azogues y (2) en la Institución Educativo Andrés Guritave se les aplico el test a tres instituciones educativas.

El test estuvo compuesto por los siguientes componentes fundamentales: (1) El primer componente evaluó la lectura y uso de inglés de manera conjunta, (2) el segundo componente se centró en la escritura, (3) el tercero midió la comprensión auditiva de los participantes y (4) el cuarto la destreza del habla del idioma. Teniendo en cuenta que los estudiantes que 
participaron en esta investigación estuvieron disponibles únicamente por una hora y 30 minutos, se modificó el test, de lo contrario no se hubiera podido evaluar todos los componentes anteriormente mencionados. El puntaje final asignado al test fue de 400 puntos significando el $100 \%$.

\subsection{Proceso de estudio}

Para el estudio, los participantes tuvieron que alcanzar en el test un puntaje de al menos 240 puntos de 400 (60\% de 100\%); la obtención de este puntaje significaría que los estudiantes poseen un nivel B1.1. según el MCER. De acuerdo con la $\mathrm{DNCl}$, los estudiantes del segundo año de bachillerato deben acreditar un nivel B1.1. Por lo tanto, este fue el nivel que evaluó el test aplicado, tomando en cuenta que los estudiantes o participantes del estudio fueron promovidos del segundo año de bachillerato al tercer año de bachillerato el año escolar anterior a la aplicación del test. Es decir, la población del estudio se encontraba cursando el tercer año de bachillerato cuando el test fue administrado. Respecto al tratamiento de los resultados del test, se realizó el siguiente procedimiento:

1. Se les entregó a los estudiantes una hoja de consentimiento para la participación en la investigación.

2. Se evaluó a los estudiantes en el tercer año de bachillerato de las unidades educativas públicas de todas las parroquias rurales del cantón Azogues, Provincia del Cañar. La logística y la movilización de los estudiantes, quienes realizaron el test, estuvo a cargo de la UNAE.

3. El instituto de Idiomas de la ESPE fue la institución encargada de la administración del test durante dos días.

4. Los resultados del test fueron entregados en una tabla Excel por la ESPE sin ningún tipo de análisis estadístico. Para propósitos de análisis, el puntaje asignado a cada competencia lingüística que midió el test fue de 100 puntos, lo cual es detallado en la Tabla 1 y Figura 1. 
5. Los datos fueron analizados por docentes- investigadores de la UNAE (autores del artículo). Todo este análisis se realizó de acuerdo con las preguntas de investigación del estudio.

\section{Resultados}

Para el análisis de los puntajes o resultados del test de los participantes se utilizó Microsoft Excel. Se hizo uso de la estadística descriptiva, específicamente las medidas de tendencia central con el propósito de determinar el dominio del idioma inglés en los cuatro componentes de dominio.

Como se indicó anteriormente, la muestra fue de 142 estudiantes y esto corresponde casi al $88.75 \%$ de la población completa, lo cual representa una muestra significativa.

A continuación, se presenta información clave sobre las instituciones educativas que participaron en la investigación.

Tabla 1. Promedio de calificaciones por unidades educativas.

\begin{tabular}{lrrrr}
\hline Unidades Educativas & $\begin{array}{c}\text { USE OF } \\
\text { ENG. \& } \\
\text { READING }\end{array}$ & LISTENING & WRITING & SPEAKING \\
\hline Unidad Educativa A & 32,37 & 17,23 & 5,63 & 45,70 \\
Unidad Educativa B & 34,68 & 3,06 & 18,18 & 69,1 \\
Unidad Educativa C & 31,39 & 21,53 & 6,13 & 33,5 \\
Unidad Educativa D & 20,4 & 16,53 & 3,47 & 35 \\
Unidad Educativa E & 28,88 & 20,54 & 8,59 & 51,1 \\
Unidad Educativa F & 34,08 & 13,76 & 0,00 & 45 \\
Unidad Educativa G & 30,71 & 13,3 & 1,7 & 60 \\
Unidad Educativa H & 31,2 & 15,38 & 0,54 & 20,4 \\
\hline
\end{tabular}

Fuente: Ortega D., y Fernández R. (2017).

La tabla 1, muestra el resultado promedio de las puntuaciones obtenidas por los estudiantes de inglés, en el tercero de bachillerato, del cantón Azogues de cada una de las ocho instituciones educativas que 
participaron en la investigación, referente a los cuatro dominios lingüísticos evaluados por el test. A su vez, muestra el valor en puntaje de cada uno de estos dominios y cuanto alcanzó cada institución.

Figura 1. Promedios por Unidades Educativas.

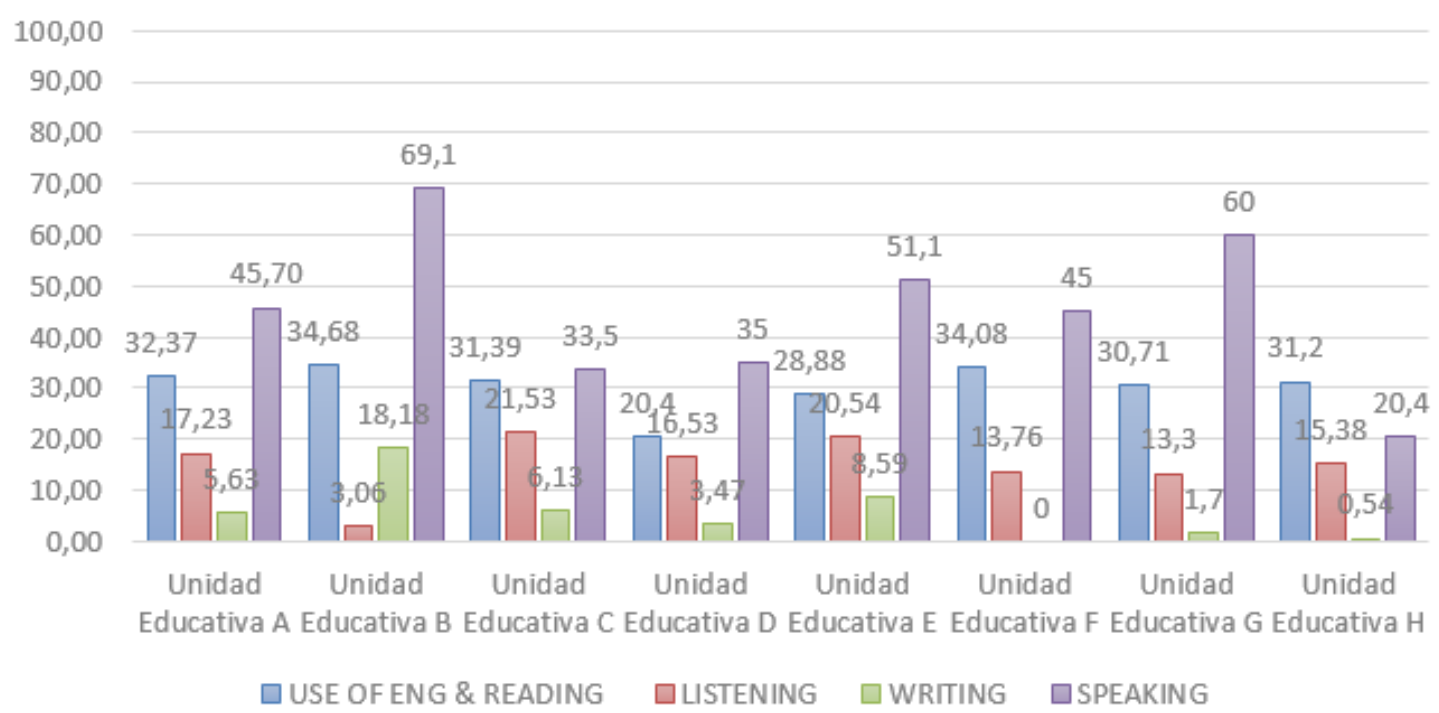

Fuente: Ortega D., y Fernández R. (2017).

La figura 1, muestra el diagrama de barras de los promedios de los puntajes obtenidos. Los estudiantes en tercero de bachillerato de la Unidad Educativa (U.E.) B lograron mayor puntaje en el componente lingüístico "speaking" saliendo con un promedio de $69.1 \%$ frente a las otras instituciones. En este mismo componente, el promedio más bajo fue de $20.4 \%$ para la U.E. $\mathrm{H}$.

A su vez, en la destreza que menos promedio obtuvieron los participantes fue en el componente lingüístico de la escritura, existiendo un $0 \%$ para la U.E. F. A pesar de este resultado existe un porcentaje de $18.18 \%$ para la U.E. B. 
En la destreza de escuchar en inglés se obtuvo un promedio de 21.53 $\%$ como puntaje mayor de la U.E. C, y el promedio de puntaje más bajo es de $3.06 \%$ para la U.E. B.

Así como, en la destreza de uso del inglés y lectura solo se alcanzó un porciento de $34.68 \%$, lo cual se le atribuye a la U.E. B, y el promedio de puntaje más bajo es de $20.4 \%$ para la U.E. D. El porcentaje de promedio de esta destreza es superado solo por la competencia lingüística de "speaking".

\section{Conclusiones y Recomendaciones}

\subsection{Conclusiones}

El test permitió corroborar las estadísticas relacionadas al bajo nivel de suficiencia del idioma inglés en el Ecuador; los resultados originales obtenidos a través de esta investigación indican que el nivel de dominio en esta lengua extranjera por parte de los estudiantes de instituciones educativas públicas de las zonas o parroquias rurales es deficiente. Este resultado nos pone de manifiesto de que las políticas educativas referentes a la enseñanza del idioma inglés en las instituciones educativas públicas todavía no han sido suficientemente óptimas para que los estudiantes que culminan la secundaria logren el nivel de dominio de inglés (B1) establecido por el MinEduc.

Esta investigación demuestra que de manera general las competencias lingüísticas del idioma inglés de los participantes del estudio son muy limitadas, especialmente la competencia lingüística productiva de la escritura y de la comprensión auditiva. En cuanto a la competencia lingüística oral, cinco de las ocho instituciones educativas tiene un promedio de porcentaje entre $20.4 \%$ y $45.70 \%$, a pesar de que una instrucción basada en metodologías comunicativas (y contemporáneas) de enseñanza de idioma extranjero deben tener un papel central en el aula de clase, según lo dispuesto en las iniciativas y reformas curriculares de la asignatura de inglés de las dos últimas décadas. 
Debido a que el dominio de la competencia de escritura está por debajo de la competencia oral en todas las unidades educativas, esto sugiere que existe una falta de énfasis en actividades de escritura que vayan más allá de una elaboración o redacción de oraciones cortas aisladas, lo cual no ayuda a los estudiantes a tener una práctica significativa en temas claves (e.g., estructuración de párrafos) de la escritura académica básica en inglés.

Además, el bajo nivel de dominio en la competencia lingüística de la comprensión auditiva entre los estudiantes dificultaría su involucramiento y participación en situaciones o propósitos comunicativos reales y auténticos que se llevan a cabo afuera del contexto de instrucción formal de las instituciones educativas; por lo tanto, esta deficiencia podría impedir a los estudiantes interactuar de manera efectiva y espontanea en el idioma meta.

El nivel de suficiencia en la competencia de lectura es más alentador, lo cual representa una fortaleza en los estudiantes de inglés de todas las unidades educativas; este aspecto positivo no solo debe ser reforzado si no también utilizado como vehículo para el desarrollo adecuado del resto de competencias.

\subsection{Recomendaciones}

Los resultados obtenidos sugieren que se debe reforzar la enseñanza de las destrezas principales del idioma inglés - leer, escribir, hablar y escuchar. En este sentido, los profesores deberían implementar estrategias y técnicas interactivas, las cuales se fundamenten en enfoques metodológicos comunicativos y contemporáneos de enseñanza de lenguas extranjeras, tales como Task-Based Language Teaching (TBLT), Communicative Language Teaching (CLT), y Content and Language Integrated Learning (CLIL).

La implementación de un enfoque metodológico integral para el desarrollo de las diferentes competencias (integrated-skills approach como se 
lo conoce en inglés) seria esencial que sea tomado en cuanto al momento de planificar y enseñar las lecciones en la clase de inglés.

El Currículo de Inglés vigente se sustenta en varios principios esenciales (e.g., aprendizaje centrado en el estudiante, desarrollo de destrezas de pensamiento, enseñanza basada en contenidos, etc.), los cuales deben ser llevados a la práctica en el aula de clase. Para implementar aspectos teóricos o conceptuales claves en la práctica docente diaria de manera efectiva, el MinEduc debo realizar capacitaciones (continuas) con el apoyo de las universidades; por ejemplo, este tipo de medida podría tener resultados positivos y representar un apoyo a los objetivos establecidos en cuanto al nivel de suficiencia que se espera que los estudiantes, al terminar el BGU, alcancen.

Estudios que exploren y examinen temas referentes al proceso de enseñanza-aprendizaje del inglés en el contexto educativo ecuatoriano deberían ser llevados a cabo en EGB y BGU. Esto permitiría conocer la realidad de este proceso en su contexto natural y especifico. Consecuentemente, ello ayudaría a generar medidas y acciones que apunten a mejorar la adquisición del idioma ingles de los estudiantes de instituciones educativas públicas de sectores urbanos y rurales, teniendo en cuenta las particularidades del sistema educativo ecuatoriano y de las necesidades específicas de los estudiantes. Así mismo, seria esencial llevar a cabo estudios que determinen y aborden las causas posibles del bajo nivel de suficiencia del idioma inglés y el bajo nivel de dominio en las competencias lingüísticas examinadas en esta investigación.

\section{Referencias}

Abreu, A. (2017). La Ética en la Investigación Educativa. Revista Scientific, 2(4), 338-350. Recuperado de:

https://doi.org/10.29394/scientific.issn.2542-2987.2017.2.4.19.338-350 
Avila, K. (2010). La instrucción guiada en la motivación, aprendizaje y dominio del idioma inglés como segunda lengua a través de las hojas de trabajo basadas en el método Kumon. Tesis de Postgrado, págs. 167. Ecuador: Universidad San Francisco de Quito. Recuperado de: http://repositorio.usfq.edu.ec/bitstream/23000/243/1/95138.pdf

British Council (2015). English in Ecuador. Quito: British Council, págs. 57. Calderón, A. (2015ab). Situación de la Educación Rural en el Ecuador. Quito: FIDA, págs. 58.

Calle, M., Calle, S., Argudo, J., Moscoso, E., Smith, A., \& Cabrera, P. (2012). Los Profesores de Inglés y su práctica docente: Un estudio de caso de los colegios fiscales de la ciudad de Cuenca, Ecuador. Maskana, 3(2), 1-17.

Creswell, J. W. (2009). Research design. Qualitative, Quantitative, and Mixed Methods Approaches (3ra Ed.). USA: Sage Publications, Inc, págs. 342.

Education First (2015). El ranking mundial más grande según su dominio del inglés, págs. 1. Recuperado de: http://www.ef.com.ec/epi/

El Ciudadano (2015). Go teacher, una cadena de excelencia para mejorar la enseñanza del inglés, págs. 1. Recuperado de:

http://www.elciudadano.gob.ec/53894/

El Comercio (2016). 3.000 estudiantes aprobaron la evaluación de inglés en la Universidad Central, págs. 1. Recuperado de:

http://www.elcomercio.com/actualidad/estudiantes-evaluacion-inglesuniversidadcentral.html

El Telégrafo (2014a). Go Teacher entrega el 12\% de docentes de inglés, págs. 1. Recuperado de:

http://www.eltelegrafo.com.ec/noticias/sociedad/4/go-teacher-entregael-12-de-docentes-de-ingles-enlace 
El Telégrafo (2014b). Ecuador está entre los países con el nivel más bajo de inglés, págs. 1. Recuperado de:

http://www.eltelegrafo.com.ec/noticias/sociedad/4/ecuador-esta-entrelos-paises-con-el-nivel-mas-bajo-de-ingles

Hernández, R., Fernández, C., Baptista, P. (2014). Metodología de la Investigación (6ta Ed.). México: McGraw-Hill, págs. 656.

Ministerio de Educación del Ecuador - MinEduc (2016). Acuerdo nro. MINEDUC-ME-2016-00020-A. Quito, Ecuador: Autor, págs. 7.

Ministerio de Educación del Ecuador - MinEduc (2009). El proyecto CRADLE: Informe final. Quito, Ecuador: Autor, págs. 40.

Ministerio de Educación del Ecuador - MinEduc (2012). National curriculum specifications: English as a foreign language. Quito, Ecuador: Autor, págs. 34.

Staehr, D., \& Kuhlman, N. (2010). Preparing teachers of English language learners: Practical Applications of the pre k-12 TESOL professional standards, págs. 12. Recuperado de:

http://www.tesol.org/docs/books/bk preparingeffectiveteachers 738

Ureña, N. (2014). English education in the Ecuadorian public sector: Gaps y recommendations, págs. 13. Recuperado de: http://repositorio.educacionsuperior.gob.ec/bitstream/28000/1404/1/TSENESCYT-00555.pdf 


\section{Diego Patricio Ortega Auquilla}

e-mail: dipaortega@gmail.com

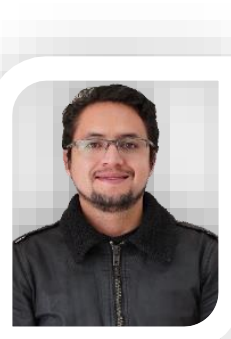

Nacido en Cuenca, Ecuador. Tiene una licenciatura en Ciencias de la Educación, especialidad en Lengua y Literatura Inglesa por la Universidad de Cuenca, Ecuador. También, realizó estudios de pregrado en Hiram College, Ohio, USA. Ha realizado cursos de nivel de posgrado en Lingüística y Didáctica de la Enseñanza de Idioma Extranjero en la Universidad Central del Ecuador. En 2014, obtuvo un máster en Currículo e Instrucción, mención en Enseñanza del Inglés en Kansas State University. Desde el año 2010 ha sido profesor en varias instituciones de educación superior. Actualmente es profesor de inglés como lengua extranjera y docente-investigador de la Universidad Nacional de Educación (UNAE) en Ecuador. 
Roxana Auccahuallpa Fernández

e-mail: roxana.auccahuallpa@unae.edu.ec

Nacida en Cusco, Perú. Tiene una Licenciatura en

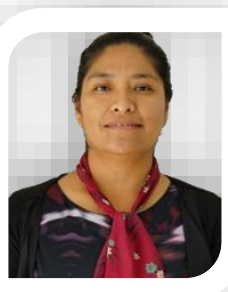

Matemática, una Maestría en Matemática Pura en la

Universidad de Puerto Rico, Recinto de Mayagüez. En

el 2014 culminó sus estudios doctorales de Currículo y

Enseñanza en Matemáticas de la Universidad de

Puerto Rico, Recinto de Río Piedras. Trabajó en Puerto

Rico en MYTI (Maximizing Yield Through Integration I) de la Universidad de Puerto Rico. Actual miembro del SEDEM Ecuador e investigador del CANP5 (Capacity and Network Project - Ecuador, Perú, Bolivia y Paraguay). Es docente de la Universidad Nacional de Educación en el Ecuador y participa en proyectos de investigación.

El contenido de este manuscrito se difunde bajo una Licencia de Creative Commons ReconocimientoNoComercial-Compartirlgual 4.0 Internacional 